\title{
Outcome of surgical approaches to the hypoplastic left heart syndrome
}

\author{
Rami Dhillon and Andrew Redington
}

\section{$\mathrm{T}$} HE HYPOPLASTIC LEFT HEART SYNDROME can be defined as a right ventriculardependent circulation associated with atresia or severe hypoplasia of the aortic valve. ${ }^{1}$ Untreated, it is uniformly fatal with more than $95 \%$ of deaths occurring in the neonatal period. ${ }^{2}$ Indeed, before the emergence of surgical therapy, it was responsible for nearly a quarter of deaths in newborns with congenital heart disease. ${ }^{3}$ Fourchamber screening is now routinely performed during antenatal ultrasound examinations, and in-utero diagnosis of hypoplastic left heart syndrome can be made by the non-cardiologist. Most parents who learn the diagnosis before 20 weeks gestation will select termination of pregnancy and this has resulted in a decline in the number of newborns with hypoplastic left heart syndrome. ${ }^{4,5}$ Despite this, the majority of cases are still encountered postnatally.

The management of this condition presents the clinician and parents with difficult practical and ethical decisions. Currently, the three postnatal therapeutic options comprise staged palliation (the Norwood protocol), 6,7 cardiac transplantation $^{8}$ or passive euthanasia. Both surgical treatment modalities are palliative and their long-term outcome is unknown. Intermediate follow-up suggests that for staged palliation, the mortality in single institution series relating to the first half of this decade ranges from 41-50\%.1,9-12 Most of the deaths occurred in the early post-operative period ( $<30$ days) following the initial Norwood palliation. A retrospective computer-based series presenting the results of neonatal admissions from 1989-1993, to a consortium of 40 university hospitals in the USA, reported an operative mortality of $53 \% .^{13}$

Accepted for publication 9 April 1997
Cardiac transplantation has generally resulted in a lower early mortality. The same United States consortium data indicated an operative mortality of $42 \%$, although the ages at which the transplants were performed, and whether any of the patients had previously undergone a staged palliation, are not clear. In a centre utilising both staged palliation and transplantation, in newborns operated $1989-1995$, the mortality was $24 \%$ at one year. ${ }^{11}$ From the Loma Linda experience, with entry into their transplant protocol during 1985-1995, the mortality at five years was $24 \% .^{8}$

Although the mortality for cardiac transplantation appears to be lower than that for staged palliation, this must be viewed in the context of limited availability of suitable donors for neonatal transplantation. In 1995, the median waiting time for infants listed for cardiac transplantation in the USA was 53 days. ${ }^{13}$ It has been recommended that in the event of a donor being unavailable by the age of 30 days, elective crossover to staged palliation occurs even though the survival of this approach is poor, as the results of transplantation for hypoplastic left heart syndrome after this time are substantially worse in most centres. ${ }^{11}$ Suitable donors are even harder to come by in the UK. To date, there have been 19 cardiac transplants performed as a primary treatment for hypoplastic left heart syndrome in the UK, of which two have survived (personal communication, transplant co-ordinators, Harefield Hospital, London; Great Ormond Street Hospital, London and Freeman Hospital, Newcastle-upon-Tyne).

The morbidity of these surgical approaches has come under closer scrutiny in recent years. Following transplantation, survivors are committed to life long immunosuppression with all its attendant risks. Chronic systemic hypertension is common $(40-81 \%)^{11,14}$ and premature coronary artery disease is beginning to be reported. ${ }^{15}$ In the 
Loma Linda series, there was a gratifyingly low incidence of mental and psychomotor delay as assessed by the Bayley scales of infant development. Neurological deficit was found in $10 \%$ of patients, mainly dystonia. ${ }^{8}$

In the survivors of staged palliation, neuropsychiatric morbidity is even more common. Hagemo and colleagues ${ }^{16}$ present in this issue of the Journal the Norwegian national experience following completion of staged palliation. The complete cohort underwent surgery overseas, by the same experienced surgical team. The $52 \%$ mortality is comparable to other series. What is striking, however, is the high prevalence of neurodevelopmental and psychological abnormalities, despite good haemodynamic results. Of the 10 patients assessed, only three had normal gross and fine motor function. Four patients first walked after 18 months. Only one of seven children (for whom this test was appropriate) could stand on one foot. Four of the 10 children were abnormal on the basis of cognitive tests. Six had problems with attention. The researchers assessed only three children (30\%) as having normal neurological and psychological function. While these results are not definitive, they are cause for concern.

Furthermore, Hagemo's results are comparable to those of Rogers et al..$^{9}$ In their series, there were 11 survivors of staged palliation, from three centres. Seven had completed the Norwood protocol, two had passed stage two, and two had had the initial operation. Only one patient was subsequently assessed as being neurodevelopmentally normal and this patient had substantial functional disability. Eight children (73\%) had acquired microcephaly (head circumference $<5$ th centile). Cognitive development was closely associated with head growth; eight of nine patients with cognitive delay had acquired microcephaly. Hagemo et al reported only three of ten children to have microcephaly but their criteria were more stringent (head circumference $<2.5$ th centile). These data should not be surprising. Seven years ago, a post mortem study detected microcephaly (head circumference <2nd centile) in $36 \%$ of babies with hypoplastic left heart syndrome, and of perhaps even more concern, $29 \%$ of 41 patients had structural malformations of the brain (absence of the corpus callosum, holoprosencephaly and cortical mantle abnormalities). ${ }^{17}$

Although these studies have small patient numbers, they do suggest that survivors of staged palliation for hypoplastic left heart syndrome have substantial neuropsychiatric morbidity, even in the presence of good haemodynamic results.
Survivors of cardiac transplantation seem to exhibit fewer neurological problems, which are chiefly motor, with relatively little cognitive dysfunction. $^{8}$ It must be noted, however, that these patients have not been exposed to the detailed neurodevelopmental scrutiny of Hagemo, Rogers and colleagues. ${ }^{?}$

It is worth re-emphasising that the currently available data can only be regarded as intermediate, at best. Increasing concerns regarding the long-term viability of the Fontan circulation, even when performed under ideal circumstances must add to the uncertainty with which we advise the parents of our patients. Can we honestly be optimistic that it will be anything other than an exceptional case who will survive into middle age with a reasonable quality of life?

Some clinicians have suggested that it is no longer ethical to abstain from a surgical treatment for patients with hypoplastic left heart syndrome. ${ }^{18}$ The results presented by Hagemo, Rogers and others must prompt us to ask whether staged palliation should be performed at all. At the very least, it is our responsibility to present the facts to parents as they exist today, rather than what we would hope them to be in the future. Whilst we await the results of larger patient cohorts, babies undergoing surgical therapy for hypoplastic left heart syndrome appear to have an uncertain neurodevelopmental outlook, even in those cases with currently satisfactory haemodynamic results.

Department of Paediatric Cardiology Royal Brompton Hospital Sydney Street London,UK

\section{References}

1. Bove EL, Lloyd TR.-year institutional experience with palliative surgery for hypoplastic left heart syndrome. Circulation 1995; 92[supp II]:II262-II266.

2. Fyler DC, Rothman KJ, Buckley LP, Cohn HE, Hellenbrand WE, Castaneda A. The determinants of five year survival of infants with critical congenital heart disease. In: Pediatric Cardiovascular Disease, Cardiovascular Clinıcs, edited by Engle MA.

3. Waston DG, Rowe RD. Aortic valve atresı: report of 43 cases JAMA 1962; 179:14-18

4 Allan LD. Fetal diagnosis of fatal congential heart disease. J Heart Lung Transplant 1993; 12 S159-\$160.

5. Allan LD. Cook A, Sullivan I, Sharland GK. Changing birth prevalence of the hypoplastic left heart syndrome as a result of fetal echocardiography. Lancet 1991; 337:959-961.

6. Norwood WI, Kirklin JK, Sanders SP. Hypoplastic left heart 
syndrome. Experıence with palliatıve surgery. Am J Cardıl 1980;87-91.

7. Norwood WI, Lang P, Hansen DD. Physiologic repair of aortic atresı-hypoplastic left heart syndrome. N Engl J Med $1983 ; 308: 23-26$.

8. Razzouk AJ, Chinnock RE, Gundry SR, Johnston JK, Larsen RL, Baum MF, Mulla NF, Bailey LL. Transplantation as a primary treatment for hypoplastic left heart syndrome: intermediate-term results. Ann Thorac Surg 1996; 62:1-8

9. Rogers BT, Msall ME, Buck GM, Lyon NR, Norris JMA, Gingell RL, Cleveland DC, Pieroni DR. Neurodevelopment outcome of infants with hypoplastic left heart syndrome. J Pediatr 1995; 126:496-498.

10. Bu'Lock FA, Stumper O, Japtag R, Silove ED, De Giovanni JV, Wright JGC, Sethia B, Brawn WJ. Surgery for infants with a hypoplastic systemic ventricle and severe outflow obstruction: early results with a modified Norwood procedure. Br Heart J 1995; 73:456-461.

11. Bando K, Turrentine MW, Sun K, Sharp TG, Caldwell RL, Darragh RK, Ensing GJ, Cordes TM, Flaspohler $\tau$, Brown $J W$. Surgical management of hypoplasia left heart syndrome. Ann Thorac Surg 1996; 62:70-77

12. Forbess JM, Cook N, Roth SJ, Seffaf A, Mayer JE, Jonas RA, Ten-year institutional experience with palliative surgery for hypoplastic left heart syndrome in a consortum of university hospitals. Am J Cardiol 1995; 76.809-811.

13. Gutgesell HP, Massaro TA. Management of hypoplastic left heart syndrome in a consortuum of university hospitals. Am J Cardiol 1995; 76. 809-811.

14. Olıvarı MT, Kubo SH, Braunlin EA, Bolman RM, Ring WS. Five-year experience with triple-drug immunosuppressive therapy in cardiac transplantation. Circulation 1990;82 (5 Suppl): IV276-280.

15. Braunlin EA, Hunter DW, Canter CE, Gutıerrez FR, Ring WS, Olivari MT, Titus JL, Spray TL, Bolman RM 3rd. Coronary artery disease in paediatric cardiac transplant recipients receiving triple-drug immunosuppression. Circulation 1991; 84 (5 Suppl): III303-309.

16. Hagemo PS, Rasmussen M, Bryhn G, Vandvik IH. Hypoplastic left heart syndrome multiprofessional follow-up in the mid-term following palliative procedures. Cardiol Young 1997; 7: 248-253

17. Glauser TA, Rorke LB, Weinberg PM, Clancy RR. Congenital brain anomalies associated with the hypoplastic left heart syndrome. Pediatrics 1990; 85: 984-990.

18. Barber $G$ Hypoplastic left heart syndrome. In: Garson A Jr, Bricker JT, McNamara DG (eds). The science and practice of pedıatric cardiology, Vol. 2. Lea and Febıger, Philadelphıa/ London 1990, pp. 1316-1333. 